\title{
Anestesia congênita de córnea associada à anestesia de ramo do trigêmio: relato de caso
}

\author{
Congenital corneal anesthesia related to trigeminal anesthesia: case report
}

\author{
Miguel José Calix Netto ${ }^{1}$ \\ Eduardo Della Giustina ${ }^{2}$ \\ Gabriel Zatti Ramos ${ }^{3}$ \\ Sheila Paula Mocellin ${ }^{4}$ \\ Luciene Barbosa de Sousa ${ }^{5}$
}

\begin{tabular}{|l|}
\hline RESUMO \\
\hline A anestesia corneana por ser uma condição rara, freqüentemente é \\
confundida ou não diagnosticada durante o exame de rotina do segmento \\
anterior. Relato de caso de um paciente de 18 anos encaminhado ao \\
ambulatório de córnea e doenças externas com quadro clinico de síndrome \\
de olho seco e com diagnóstico provável de síndrome de Sjögren. Era \\
amblíope de olho direito devido à opacidade corneana no eixo visual \\
secundária a trauma com unha na infância. Foi pesquisada sensibilidade \\
corneana que era ausente em ambos os olhos; olho seco grave e com BUT \\
(tempo de quebra do filme lacrimal) menor que 4 segundos. Foi feito \\
diagnóstico de anestesia corneana congênita associada a hipoestesia do \\
nervo trigêmio pela avaliação neurológica da sensibilidade facial e \\
movimentos bruscos do queixo que evidenciavam alterações sensoriais \\
do nervo. O oftalmologista geral e principalmente o especialista em \\
segmento anterior devem ter como rotina a pesquisa da sensibilidade \\
corneana no exame do segmento anterior.
\end{tabular}

Descritores: Doenças da córnea/congênito; Doenças da córnea/diagnóstico; Córnea/ inervação; Hipoestesia/congênito; Doenças do sistema nervoso autônomo; Técnicas de diagnóstico em Oftalmologia; Relatos de casos [Tipo de Publicação]

\section{INTRODUÇÃO}

A anestesia corneana congênita é uma entidade bem definida, porém pouco diagnosticada devido à falta de suspeita clínica e por tratar-se de um diagnóstico de exclusão. Em sua entidade isolada, pode ser unilateral (menos comum) ou bilateral ${ }^{(1-2)}$; já associada a uma síndrome tem sido descrita na insensibilidade congênita; a dor, na síndrome de Riley-Day, ambas classificadas como neuropatias sensoriais autônomas, Goldenhar-Gorlin e MURCS (aplasia do ducto mulleriano, aplasia renal e displasia dos somitos cervicotorácicos $)^{(3-4)}$. Freqüentemente é confundida com ceratite herpética, erosão corneana recorrente e olho seco, pois todas podem fazer parte do quadro clínico ${ }^{(5)}$. As opacidades corneanas, ceratite e erosão corneana recorrente na infância, algumas vezes acompanhadas de automutilação, principalmente corneana, podem ser o primeiro sinal de anestesia corneana congênita. O quadro clínico puramente corneano é raro, sendo mais freqüente a associação com hipoestesia na área inervada pelo nervo trigêmeo.

A anestesia congênita do nervo trigêmio afeta toda a parte sensorial do nervo trigêmio, é bilateral e associada com opacidades corneana indolores ${ }^{(6)}$. Esta condição tem sido classificada em três tipos diferentes baseada na etiologia:

Tipo 1: secundária a hipoplasia do núcleo trigeminal;

Tipo 2: associada ao desenvolvimento anormal do ectoderma e mesoderma (Möebius e Goldenhar); 
Tipo 3: associado a anormalidades focais do sistema nervoso central (paralisia de nervos cranianos) ${ }^{(6)}$.

Temos como objetivo apresentar um relato de caso de anestesia corneana bilateral associada à hipoestesia do nervo trigêmio, alertando aos oftalmologistas em geral que a pesquisa da sensibilidade corneana deve fazer parte da rotina de exame clínico do segmento anterior.

Foi avaliado um caso do ambulatório de córnea e doenças externas do Hospital Oftalmológico de Sorocaba, com anamnese completa, exame oftalmológico completo de rotina, incluindo pesquisa da sensibilidade corneana com cotonete de algodão, teste de Schimer tipo I e teste do tempo de quebra da fluoresceína, avaliação corneana com corante rosa bengala. Foram feitas fotografias digitais da pesquisa da sensibilidade corneana, bem como um filme. A paciente em estudo foi avaliada por um otorrinolaringologista do próprio hospital, e solicitados audiometria e exame de pesquisa da via neuronal auditiva (BERA). Foram também solicitadas uma avaliação neurológica da paciente e a realização de exames laboratoriais de rotina e específicos para síndrome do olho seco.

\section{RELATO DO CASO}

Paciente A.O. de 18 anos de idade, sexo feminino, natural e procedente de São Paulo, referida ao nosso Serviço em fevereiro de 2004, com queixa de embaçamento visual em olho esquerdo, acompanhado de ardência, irritação e sensação de corpo estranho em ambos os olhos. Acompanhava em outro serviço há dois anos com diagnóstico de olho seco por síndrome de Sjögren, sem melhora dos sintomas. Mãe referia que filha era deficiente auditiva e vinha apresentando baixa progressiva da acuidade visual em olho esquerdo. Informou que visão do olho direito sempre foi ruim desde pequena, devido a trauma com a própria unha (sic).

História patológica pregressa: parto cesariano, hipoacusia desde quatro meses de idade, automutilação do nariz aos seis meses, trauma de olho direito com a própria unha com sete meses de vida; pupiloplastia óptica em olho direito com um ano de idade em um hospital de referência em São Paulo (sic).

No exame oftalmológico apresentava: acuidade visual, com correção - olho direito: movimento de mão e olho esquerdo: 20/40 (-0,50DE -2,00DC a 40); pressão intra-ocular - olho direito: $14 \mathrm{mmHg}$ e olho esquerdo: $12 \mathrm{mmHg}$; biomicroscopia olho direito: opacidade corneal estromal de $3+/ 4+$, pupiloplastia óptica às $12 \mathrm{~h}$ e ceratite puntata difusa e olho esquerdo: opacidade corneana central $1+/ 4+$, degeneração tipo Salzman e ceratite puntata difusa. Sensibilidade corneana ausente em ambos os olhos. BUT (tempo de quebra da fluoresceína) menor que 4 segundos em ambos os olhos e teste de Rosa Bengala utilizando a escala de Bjsterveld em ambos os olhos: terço temporal $=1$; terço central $=2$; terço nasal $=3$. Teste de Schirmer tipo I avaliando a secreção basal e reflexa em ambos os olhos: menor que $5 \mathrm{~mm}$. Fundoscopia em ambos os olhos: sem alterações. Exame de sensibilidade facial alterada em área inervada pelo nervo trigêmeo, tônus do músculo masseter à palpação diminuído e movimento brusco do queixo normal.

Exames complementares - A paciente já possuía em mãos os seguintes exames laboratoriais: Hemograma, glicemia jejum, PPD, VDRL, FTA-ABS IgG e IgM, Anti-ssb (la), fator reumatóide, TORCH, pesquisa de anticorpos anti-herpes simples vírus dentro dos limites da normalidade. VHS $1 \mathrm{~h}$ e $2 \mathrm{~h}$ elevadas para o sexo e idade. Radiografia de coluna revelava pelo laudo: escoliose tipo S dorso lombar; tomografias computadorizadas de crânio, órbitas, cócleas e canal auditivo sem alterações. O laudo da audiometria acusou: hipoacusia profunda bilateral e reflexos acústicos ausentes. O exame BERA evidenciou que a via auditiva (neuronal) estava preservada.

Com isso o tratamento, baseado na síndrome do olho seco seguiu a seguinte sequiência: lubrificação e controle de fatores externos como ar condicionado; uso de umidificadores de ambiente; oclusão de pontos lacrimais em ambos os olhos que de inicio foi temporária e não sendo suficiente, evolui com oclusão definitiva; tarsorrafia definitiva de terço temporal.

A paciente evoluiu satisfatoriamente referindo melhora sintomática e da acuidade visual do olho esquerdo.

Ao exame da acuidade visual com correção: o olho direito permaneceu em movimento de mão e do olho esquerdo evoluiu para 20/25. Houve melhora da transparência corneana à biomicroscopia principalmente de olho esquerdo, o BUT em ambos os olhos evoluiu para 7 segundos e o teste de Schirmer tipo I passou para $5 \mathrm{~mm}$ em ambos os olhos.

\section{DISCUSSÃO}

A córnea é ricamente inervada por fibras do gânglio do nervo trigêmeo (ramo oftálmico), responsável pela sensibilidade e com isso proteção desse órgão ocular nobre.

Apresentamos um caso de olho seco em paciente jovem de 18 anos secundário a deficiência da produção aquosa por perda do reflexo de lacrimejamento e com história de automutilação. O caso trata-se provavelmente de anestesia congênita de córnea secundária à anestesia de ramo do trigêmeo, pois apresenta área de hipoestesia relacionada à inervação do referido nervo, bem como diminuição do tônus muscular do masseter que é inervado pelas fibras mandibulares do trigêmeo; anestesia do ramo oftálmico que inerva a córnea. A grande maioria dos casos de hipoestesia ou anestesia corneana não se restringe ao comprometimento isolado da mesma e apresenta-se mais freqüentemente associado com alterações da musculatura inervada pelo nervo trigêmeo. Em países subdesenvolvidos, a deficiência de vitamina A e a hanseníase devem ser afastadas e entram no diagnóstico diferencial ${ }^{(7)}$. Afastamos síndrome de Riley-Day por não apresentar alteração no sistema nervoso autônomo e ser mais freqüente a um tipo étnico restrito. A paciente em estudo apresentou historia de auto-mutilação que pode estar associado ao quadro de hipoestesia corneana congênita secundária a anestesia de ramo de trigêmeo. Apresenta também hipoacusia que pode 
estar relacionada a uma doença sistêmica ou síndrome ainda em pesquisa. Concluímos que, para um correto diagnóstico de olho seco, deve-se incluir o teste de sensibilidade corneana devendo este fazer parte do exame de rotina do segmento anterior que, neste caso, nos levou ao diagnóstico e conduta adequados. O prognóstico final nestes casos depende da alta suspeição clínica e a pesquisa de rotina da sensibilidade corneana para o diagnóstico precoce e correto, impedindo com isso as complicações como opacidade corneana, perfuração e perda visual.

\section{ABSTRACT}

Corneal anesthesia is a rare condition, therefore its diagnosis is frequently impaired or it is not noticed during the anterior segment examination. Case report of a 18-year-old patient referred to our Corneal and External Disease Department who complained of dry eye symptoms and with a suspicion of Sjögren's syndrome. She had amblyopia of the right eye, consequence of corneal leucoma over the visual axis secondary to a fingernail traumatism inflicted by herself in childhood. On the ophthalmologic examination corneal sensitivity was absent in both eyes. Severe dry eye and breakup time less than four seconds. Diagnosis of congenital corneal anesthesia was established, secondary to trigeminal anesthesia found on neurological evaluation of facial sensitivity. She also showed sudden movements of the chin which evidenced sensorial pathology of the trigeminal nerve. The general ophthalmologist and specially anterior segment specialists must perform tests for corneal sensitivity during the routine eye examination.

Keywords: Corneal diseases/congenital; Corneal diseases/ diagnosis; Cornea/innervation; Hypesthesia/congenital; Autonomic nervous system diseases; Diagnostic techniques, ophthalmological; Case reports [Publication type]

\section{REFERÊNCIAS}

1. Miller NR. Anatomy and physiology of trigeminal nerve. In: Tansill BC, editor. Walsh and Hoyt's clinical neuro-ophthalmology. Baltimore: Williams \& Wilkins; 1985. p.999-1070.

2. Stewart HL, Wind CA, Kaufman HE. Unilateral congenital corneal anesthesia. Am J Ophthalmol. 1972;74(2):334-5.

3. Mohandessan MM, Romano PE. Neuroparalytic keratitis in Goldenhar-Gorlin syndrome. Am J Ophthalmol. 1978;85(1):111-3.

4. Esakowitz L, Yates JR. Congenital corneal anaesthesia and the MURCS association: a case report. Br J Ophthalmol. 1988;72(3):236-8.

5. Mathen MM, Vishnu S, Prajna NV, Vijayalakshmi P, Srinivasan M. Congenital corneal anesthesia: a series of four case reports. Cornea. 2001;20(2):194-6.

6. Wong VA, Cline RA, Dubord PJ, Rees M. Congenital trigeminal anesthesia in two siblings and their long-term follow-up. Am J Ophthalmol. 2000;129(1): 96-8.

7. Krishnamoorthy KV. Congenital absence of sensation: its differential diagnosis. Lepr India. 1982;54(3):499-504 